\title{
A Non-Preemptive Priority Queueing System with a Single Server Serving Two Queues M/G/1 and M/D/1 with Optional Server Vacations Based on Exhaustive Service of the Priority Units
}

\author{
Kailash C. Madan \\ College of Information Technology, Ahlia University, Manama, Kingdom of Bahrain \\ E-mail:kcmadan@yahoo.com,kailash@ahliauniversity.edu.bh \\ Received March 22, 2011; revised May 9, 2011; accepted May 13, 2011
}

\begin{abstract}
We study a vacation queueing system with a single server simultaneously dealing with an $\mathrm{M} / \mathrm{G} / 1$ and an $\mathrm{M} / \mathrm{D} / 1$ queue. Two classes of units, priority and non-priority, arrive at the system in two independent Poisson streams. Under a non-preemptive priority rule, the server provides a general service to the priority units and a deterministic service to the non-priority units. We further assume that the server may take a vacation of random length just after serving the last priority unit present in the system. We obtain steady state queue size distribution at a random epoch. Corresponding results for some special cases, including the known results of the $\mathrm{M} / \mathrm{G} / 1$ and the $\mathrm{M} / \mathrm{D} / 1$ queues, have been derived.
\end{abstract}

Keywords: Non Preemptive Priority Queueing System, Modified Server Vacations, Combination of General Service and Deterministic Service, Steady State, Queue Size Distribution

\section{Introduction}

Several authors including Cobham [1], Phipps [2], Schrage [3], Jaiswal [4], Madan [5], Simon [6], Takagi [7], Choi and Chang [8] have studied priority queues. These authors and several others have studied single server or multi-server queues with two or more priority classes under preemptive or non-preemptive priority rules. All these authors essentially assume the same service time distribution for all classes of units with identical or different service rates. Madan and Abu-Dayyeh [9] deal with a single server queueing system with two classes of units, priority units and non-priority units. Under the non-preemptive queue discipline, they assume that the service time $\mathrm{V}$ of a priority unit has a general distribution and that of a non-priority unit is deterministic. Thus their model is a combination of the $\mathrm{M} / \mathrm{G} / 1$ and $\mathrm{M} / \mathrm{D} / 1$ queues and the server keeps switching over these two queues depending on the class of units present in the system. For separate references on $\mathrm{M} / \mathrm{G} / 1$ and $\mathrm{M} / \mathrm{D} / 1$ queues, the reader is referred to Bhat [10], Levy and Yechiali [11], Kleinrock [12], Cohen [13], Lee [14], Gross and Harris [5], Cox and Miller [16], Tijms [17], Yang and Li [18],
Bunday [19] and Madan [20,21]. However, in the present paper, we generalize Madan and Abu-Dayyeh [9] paper by adding a significant assumption to their model that the server may take a vacation of random length but we assume that no vacation is allowed if there is even a single priority unit present in the system. Thus the server may take an optional vacation of a random length just after completing the service of the last priority unit present in the system or else may just continue serving the non-priority units if present in the system.

We use the supplementary variable technique by introducing two supplementary variables, one for the elapsed service time of a priority unit and the other for the elapsed vacation time of the server. Thus, we generalize the results of not only Madan and Abu-Dayyeh [9], but also some other known results of the M/G/1 and the $\mathrm{M} / \mathrm{D} / 1$ queues as particular cases.

\section{Assumptions Underlying the Mathematical Model}

Priority and non-priority units arrive at the system in independent Poisson streams with respective mean arri- 
val rates $\lambda_{1}$ and $\lambda_{2}$ and form two queues, if the server is busy. The server must serve all the priority units present in the system before taking up a non-priority unit for service. In other words, there is no priority unit present in the system at the time of starting service of a nonpriority unit. Further, we assume that the server follows a non-preemptive priority rule, which means that if one or more priority units arrive during the service time of a non-priority unit, the current service of a non-priority unit is not stopped and a priority unit will be taken up for service only after the current service of a non-priority unit is complete. Units are served one by one, on a 'first-come, first-served' basis within each class of units. We assume that the service time $S$ of a priority unit is general with probability density function $b(s)$ and the distribution function $B(s)$. Let $\mu(x) \mathrm{dx}$ be the conditional probability of completion of service of a priority unit during the interval $(x, x+d x]$ given that the elapsed service time of such a unit is $x$, so that

$$
\mu(x)=\frac{b(x)}{1-B(x)}
$$

and, therefore,

$$
b(s)=\mu(s) \exp \left[-\int_{0}^{s} \mu(x) \mathrm{d} x\right] .
$$

The service time of a non-priority unit is deterministic with constant duration $d \quad(>0)$.

We further assume that as soon as the service of the last priority unit present in the system is completed, the server has the option to take a vacation of random length with probability $p$, in which case the vacation starts immediately or else with probability $(1-p)$ he may decide to continue serving the non-priorty units present in the system, if any. In the later case, if there is no non-priority unit present in the system, the server remains idle in the system waiting for the new units to arrive. The vacation period random variable $V$ is assumed to follow a general probability law with probability density function $a(v)$ and the distribution function $A(v)$. Let $\beta(x) \mathrm{d} x$ be the conditional probability of completion of server's vacation during the interval $(x, x+d x]$ given that the elapsed vacation time of the server is $x$, so that

$$
\beta(x)=\frac{a(x)}{1-A(x)}
$$

and, therefore,

$$
a(s)=\beta(s) \exp \left[-\int_{0}^{s} \beta(x) \mathrm{d} x\right]
$$

\section{Definitions and Notations}

\section{We define}

$P_{m, n}^{(1)}(x, t)$ : probability that at time $t$ there are $m(\geq 0)$ priority units and $n(\geq 0)$ non-priority units in the queue excluding one priority unit in service with elapsed service time $x$.

$$
P_{m, n}^{(1)}(t)=\int_{0}^{\infty} P_{m, n}^{(1)}(x, t) \mathrm{d} x \text { : probability that at time } t \text { there }
$$

are $\mathrm{m}(\geq 0)$ priority units and $n(\geq 0)$ non-priority units in the queue excluding one priority unit in service without regard to the elapsed service time $x$ of a priority unit.

$V_{m, n}(x, t)$ : probability that at time $t$ the server is on vacation with elapsed vacation time $x$ and there are $m$ $(\geq 0)$ priority units and $n(\geq 0)$ non-priority units in the queue.

$$
V_{m, n}(t)=\int_{0}^{\infty} V_{m, n}(x, t) \mathrm{d} x \text { : probability that at time } t \text { the }
$$

server is on vacation and there are $m(\geq 0)$ priority units and $n(\geq 0)$ non-priority units in the queue, without regard to the elapsed repair time $x$.

$P_{0, n}^{(2)}(t)$ : probability that at time $t$ there are no priority units in the system and $n(\geq 0)$ non-priority units in the queue excluding one non-priority unit in service.

$Q(t)$ : probability that at time $t$ there is neither a priority unit nor a non-priority unit in the system and the server is idle but available in the system.

$r_{i}$ : probability that $i(=0,1,2, \cdots)$ priority units arrive during the constant service time $\mathrm{d}$ of a non-priority unit.

$k_{j}$ : probability that $j(=0,1,2, \cdots)$ non-priority units arrive during the constant service time $\mathrm{d}$ of a non-priority unit.

Then assuming that the steady state exists, let

$$
\begin{gathered}
\lim _{t \rightarrow \infty} P_{m, n}^{(1)}(x, t)=P_{m, n}^{(1)}(x), \\
\lim _{t \rightarrow \infty} P_{m, n}^{(1)}(t)=\int_{0}^{\infty} P_{m, n}^{(1)}(x) \mathrm{d} x=P_{m, n}^{(1)}, \\
\lim _{t \rightarrow \infty} V_{m, n}(x, t)=V_{m, n}(x),
\end{gathered}
$$

$\lim _{t \rightarrow \infty} V_{m, n}(t)=\int_{0}^{\infty} V_{m, n}(x) \mathrm{d} x=V_{m, n} ; \quad \lim _{t \rightarrow \infty} P_{0, n}^{(2)}(t)=P_{0, n}^{(2)}$

and $\lim _{t \rightarrow \infty} Q(t)=Q$

denote the corresponding steady state probabilities. In addition, we define the following steady state probability generating functions: 


$$
\begin{gathered}
P_{m}^{(1)}\left(x, z_{2}\right)=\sum_{n=0}^{\infty} P_{m, n}^{(1)}(x) z_{2}^{n} ; P_{n}^{(1)}\left(x, z_{1}\right)=\sum_{m=0}^{\infty} P_{m, n}^{(1)}(x) z_{1}^{m}, \\
P^{(1)}\left(x, z_{1}, z_{2}\right)=\sum_{m=0}^{\infty} \sum_{n=0}^{\infty} P_{m, n}^{(1)}(x) z_{1}^{m} z_{2}^{n}=\sum_{m=0}^{\infty} P_{m}^{(1)}\left(x, z_{2}\right) z_{1}^{m}=\sum_{n=0}^{\infty} P_{n}^{(1)}\left(x, z_{1}\right) z_{2}^{n}, \\
P^{(1)}\left(z_{1}, z_{2}\right)=\int_{0}^{\infty} P^{(1)}\left(x, z_{1}, z_{2}\right) \mathrm{d} x=\sum_{m=0}^{\infty} \sum_{n=0}^{\infty} P_{m, n}^{(1)} z_{1}^{m} z_{2}^{n}, \\
V_{m}\left(x, z_{2}\right)=\sum_{n=0}^{\infty} V_{m, n}(x) z_{2}^{n} ; V_{n}\left(x, z_{1}\right)=\sum_{m=0}^{\infty} V_{m, n}(x) z_{1}^{m}, \\
V\left(x, z_{1}, z_{2}\right)=\sum_{m=0}^{\infty} \sum_{n=0}^{\infty} V_{m, n}(x) z_{1}^{m} z_{2}^{n}=\sum_{m=0}^{\infty} V_{m}\left(x, z_{2}\right) z_{1}^{m}=\sum_{n=0}^{\infty} V_{n}\left(x, z_{1}\right) z_{2}^{n}, \\
V\left(z_{1}, z_{2}\right)=\int_{0}^{\infty} V\left(x, z_{1}, z_{2}\right) \mathrm{d} x=\sum_{m=0}^{\infty} \sum_{n=0}^{\infty} V_{m, n} z_{1}^{m} z_{2}^{n}, \\
P_{0}^{(2)}\left(z_{2}\right)=\sum_{n=0}^{\infty} P_{0, n}^{(2)} z_{2}^{n}, \\
P_{0}^{(1)}\left(z_{2}\right)=\sum_{n=0}^{\infty} P_{0, n}^{(1)} z_{2}^{n}, \\
K\left(z_{2}\right)=\sum_{j=0}^{\infty} k_{j} z_{2}^{j}=\sum_{j=0}^{\infty}\left(\frac{\exp \left(-\lambda_{2} d\right)\left(\lambda_{2} d\right)^{j}}{j !} z_{i=0}^{\infty} r_{1}^{i}=\sum_{i=0}^{\infty}\left(\frac{\exp \left(-\lambda_{1} d\right)\left(\lambda_{1} d\right)^{i}}{i !}\right) z_{1}^{i}=\exp \left[-\lambda_{2} d\left(1-z_{2}\right)\right], \leq 1,\left|z_{2}\right| \leq 1 .\right. \\
\left.\mid z_{1} d\left(1-z_{1}\right)\right],
\end{gathered}
$$

\section{Steady State Equations Governing the System}

Usual probability reasoning based on our mathematical model, leads to the following equations.

$$
\begin{gathered}
\frac{\mathrm{d}}{\mathrm{d} x} P_{m, n}^{(1)}(x)+\left(\lambda_{1}+\lambda_{2}+\mu(x)\right) P_{m, n}^{(1)}(x)=\lambda_{1} P_{m-1, n}^{(1)}(x)+\lambda_{2} P_{m, n-1}^{(1)}(x), m \geq 1, n \geq 1, \\
\frac{\mathrm{d}}{\mathrm{d} x} P_{m, 0}^{(1)}(x)+\left(\lambda_{1}+\lambda_{2}+\mu(x)\right) P_{m, 0}^{(1)}(x)=\lambda_{1} P_{m-1,0}^{(1)}(x), m \geq 1, n=0, \\
\frac{\mathrm{d}}{\mathrm{d} x} P_{0, n}^{(1)}(x)+\left(\lambda_{1}+\lambda_{2}+\mu(x)\right) P_{0, n}^{(1)}(x)=\lambda_{2} P_{0, n-1}^{(1)}(x), m=0, n \geq 1, \\
\frac{\mathrm{d}}{\mathrm{d} x} P_{0,0}^{(1)}(x)+\left(\lambda_{1}+\lambda_{2}+\mu(x)\right) P_{0,0}^{(1)}(x)=0, m=0, n=0, \\
\frac{\mathrm{d}}{\mathrm{d} x} V_{m, n}(x)+\left(\lambda_{1}+\lambda_{2}+\beta(x)\right) V_{m, n}(x)=\lambda_{1} V_{m-1, n}(x)+\lambda_{2} V_{m, n-1}(x), m \geq 1, n \geq 1, \\
\frac{\mathrm{d}}{\mathrm{d} x} V_{m, 0}(x)+\left(\lambda_{1}+\lambda_{2}+\beta(x)\right) V_{m, 0}(x)=\lambda_{1} V_{m-1,0}(x), m \geq 1, n=0, \\
\frac{\mathrm{d}}{\mathrm{d} x} V_{0, n}(x)+\left(\lambda_{1}+\lambda_{2}+\beta(x)\right) V_{0, n}(x)=\lambda_{2} V_{0, n-1}(x), m=0, n \geq 1,
\end{gathered}
$$




$$
\begin{gathered}
\frac{\mathrm{d}}{\mathrm{d} x} V_{0,0}(x)+\left(\lambda_{1}+\lambda_{2}+\beta(x)\right) V_{0,0}(x)=0, m=0, n=0, \\
Q=\left(Q+P_{0,0}^{(2)}\right) r_{0} k_{0}+(1-p) \int_{0}^{\infty} P_{0,0}^{(1)} \mu(x) \mathrm{d} x+\int_{0}^{\infty} V_{0,0}(x) \beta(x) \mathrm{d} x, \\
P_{0,0}^{(2)}=\left(Q+P_{0,0}^{(2)}\right) r_{0} k_{1}+P_{0,1}^{(2)} r_{0} k_{0}+(1-p) \int_{0}^{\infty} P_{0,1}^{(1)} \mu(x) \mathrm{d} x+\int_{0}^{\infty} V_{0,1}(x) \beta(x) \mathrm{d} x, \\
P_{0, n}^{(2)}=\left(Q+P_{0,0}^{(2)}\right) r_{0} k_{n+1}+\sum_{j=1}^{n+1} P_{0, j}^{(2)} r_{0} k_{n+1-j}+(1-p) \int_{0}^{\infty} P_{0, n+1}^{(1)} \mu(x) \mathrm{d} x+\int_{0}^{\infty} V_{0, n+1}(x) \beta(x) \mathrm{d} x, n \geq 1 .
\end{gathered}
$$

The above equations are to be solved subject to the following boundary conditions:

$$
\begin{gathered}
P_{m, n}^{(1)}(0)=\int_{0}^{\infty} P_{m+1, n}^{(1)}(x) \mu(x) \mathrm{d} x+\sum_{j=0}^{n} P_{0, j}^{(2)} r_{m+1} k_{n-j}+Q r_{m+1} k_{n}+\int_{0}^{\infty} V_{m+1, n}(x) \beta(x) \mathrm{d} x, m \geq 1, n \geq 1, \\
P_{m, 0}^{(1)}(0)=\int_{0}^{\infty} P_{m+1,0}^{(1)}(x) \mu(x) \mathrm{d} x+P_{0,0}^{(2)} r_{m+1} k_{0}+Q r_{m+1} k_{0}+\int_{0}^{\infty} V_{m+1,0}(x) \beta(x) \mathrm{d} x, m \geq 1, n=0, \\
P_{0, n}^{(1)}(0)=\int_{0}^{\infty} P_{1, n}^{(1)}(x) \mu(x) \mathrm{d} x+\sum_{j=0}^{n} P_{0, j}^{(2)} r_{1} k_{n-j}+Q r_{1} k_{n}+\int_{0}^{\infty} V_{1, n}(x) \beta(x) \mathrm{d} x, m=0, n \geq 1, \\
P_{0,0}^{(1)}(0)=\int_{0}^{\infty} P_{1,0}^{(1)}(x) \mu(x) \mathrm{d} x+P_{0,0}^{(2)} r_{1} k_{0}+Q r_{1} k_{0}+\int_{0}^{\infty} V_{1,0}(x) \beta(x) \mathrm{d} x, m=0=n . \\
V_{0, n}(0)=p \int_{0}^{\infty} P_{0, n}^{(1)}(x) \mu(x) \mathrm{d} x, n \geq 0
\end{gathered}
$$

\section{Steady State Queue Size Distribution at a Random Epoch}

We perform the operations $\sum_{n=1}^{\infty}(4.1) z_{2}^{n}+(4.2) ; \sum_{n=1}^{\infty}(4.3) z_{2}^{n}+(4.4)$ and use Equation (3.1). Thus we obtain

$$
\begin{gathered}
\frac{\mathrm{d}}{\mathrm{d} x} P_{m}^{(1)}\left(x, z_{2}\right)+\left(\lambda_{1}+\lambda_{2}+\mu(x)\right) P_{m}^{(1)}\left(x, z_{2}\right)=\lambda_{1} P_{m-1}^{(1)}\left(x, z_{2}\right)+\lambda_{2} z_{2} P_{m}^{(1)}\left(x, z_{2}\right), m \geq 1, \\
\frac{\mathrm{d}}{\mathrm{d} x} P_{0}^{(1)}\left(x, z_{2}\right)+\left(\lambda_{1}+\lambda_{2}+\mu(x)\right) P_{0}^{(1)}\left(x, z_{2}\right)=\lambda_{2} z_{2} P_{0}^{(1)}\left(x, z_{2}\right) .
\end{gathered}
$$

Next, we perform $\sum_{m=1}^{\infty}(4.17) z_{1}^{m}+(4.18)$, use (3.1) and simplify. Then we have,

$$
\frac{\mathrm{d}}{\mathrm{d} x} P^{(1)}\left(x, z_{1}, z_{2}\right)+\left(\lambda_{1}\left(1-z_{1}\right)+\lambda_{2}\left(1-z_{2}\right)+\mu(x)\right) P^{(1)}\left(x, z_{1}, z_{2}\right)=0
$$

Similarly, we perform the operations $\sum_{n=1}^{\infty}(4.5) z_{2}^{n}+(4.6) ; \sum_{n=1}^{\infty}(4.7) z_{2}^{n}+(4.8)$ and use Equation (3.1). Thus we obtain

$$
\begin{gathered}
\frac{\mathrm{d}}{\mathrm{d} x} V_{m}\left(x, z_{2}\right)+ \\
\left(\lambda_{1}+\lambda_{2}+\beta(x)\right) V_{m}\left(x, z_{2}\right)=\lambda_{1} V_{m-1}\left(x, z_{2}\right)+\lambda_{2} z_{2} V_{m}\left(x, z_{2}\right), m \geq 1 \\
\frac{\mathrm{d}}{\mathrm{d} x} V_{0}\left(x, z_{2}\right)+\left(\lambda_{1}+\lambda_{2}+\beta(x)\right) V_{0}\left(x, z_{2}\right)=\lambda_{2} z_{2} V_{0}\left(x, z_{2}\right) .
\end{gathered}
$$

Next, we perform $\sum_{m=1}^{\infty}(4.20) z_{1}^{m}+(4.21)$, use (3.1) and simplify. Then we have, 


$$
\frac{\mathrm{d}}{\mathrm{d} x} V\left(x, z_{1}, z_{2}\right)+\left(\lambda_{1}\left(1-z_{1}\right)+\lambda_{2}\left(1-z_{2}\right)+\beta(x)\right) V\left(x, z_{1}, z_{2}\right)=0
$$

Then we perform $(4.9)+(4.10) z_{2}+\sum_{n=1}^{\infty}(4.11) z_{2}^{n+1}$, use (3.1) and simplify. Thus we have

$$
z_{2} P_{0}^{(2)}\left(z_{2}\right)=Q r_{0} K\left(z_{2}\right)+(1-p) \int_{0}^{\infty} P_{0}^{(1)}\left(x, z_{2}\right) \mu(x) \mathrm{d} x+P_{0}^{(2)}\left(z_{2}\right) r_{0} K\left(z_{2}\right)-Q+\int_{0}^{\infty} V_{0}\left(x, z_{2}\right) \beta(x) \mathrm{d} x,
$$

which again simplifies to

$$
\left(z_{2}-r_{0} K\left(z_{2}\right)\right) P_{0}^{(2)}\left(z_{2}\right)=(1-p) \int_{0}^{\infty} P_{0}^{(1)}\left(x, z_{2}\right) \mu(x) \mathrm{d} x+Q r_{0} K\left(z_{2}\right)-Q+\int_{0}^{\infty} V_{0}\left(x, z_{2}\right) \beta(x) \mathrm{d} x .
$$

Now, we shall consider the boundary conditions (4.12) through (4.16) and perform $\sum_{m=1}^{\infty}(4.12) z_{1}^{m+1}+(4.14) z_{1}$; $\sum_{m=1}^{\infty}(4.13) z_{1}^{m+1}+(4.15) z_{1}$, use (3.1) and simplify. We then obtain

$$
\begin{aligned}
z_{1} P_{n}^{(1)}\left(0, z_{1}\right)= & \int_{0}^{\infty} P_{n}^{(1)}\left(x, z_{1}\right) \mu(x) \mathrm{d} x-\int_{0}^{\infty} P_{0, n}^{(1)}(x) \mu(x) \mathrm{d} x+\int_{0}^{\infty} V_{n}\left(x, z_{1}\right) \beta(x) \mathrm{d} x-\int_{0}^{\infty} V_{0, n}(x) \beta(x) \mathrm{d} x \\
& +\left(R\left(z_{1}\right)-r_{0}\right) \sum_{j=0}^{n} P_{0, j}^{(2)} k_{n-j}+\left(R\left(z_{1}\right)-r_{0}\right) \sum_{n=1}^{\infty} Q k_{n}, \quad n \geq 1,
\end{aligned}
$$

$z_{1} P_{0}^{(1)}\left(0, z_{1}\right)=\int_{0}^{\infty} P_{0}^{(1)}\left(x, z_{1}\right) \mu(x) \mathrm{d} x-\int_{0}^{\infty} P_{0,0}^{(1)}(x) \mu(x) \mathrm{d} x+\int_{0}^{\infty} V_{0}\left(x, z_{1}\right) \beta(x) \mathrm{d} x-\int_{0}^{\infty} V_{0,0}(x) \beta(x) \mathrm{d} x+\left(R\left(z_{1}\right)-r_{0}\right)\left(P_{0,0}^{(2)}+Q\right) k_{0}$.

And yet again, we perform $\sum_{n=1}^{\infty}(4.25) z_{2}^{n}+(4.26)$, use (3.1) and simplify. This operation yields

$$
\begin{aligned}
z_{1} P^{(1)}\left(0, z_{1}, z_{2}\right)= & \int_{0}^{\infty} P^{(1)}\left(x, z_{1}, z_{2}\right) \mu(x) \mathrm{d} x-\int_{0}^{\infty} P_{0}^{(1)}\left(x, z_{2}\right) \mu(x) \mathrm{d} x+\int_{0}^{\infty} V\left(x, z_{1}, z_{2}\right) \beta(x) \mathrm{d} x-\int_{0}^{\infty} V_{0}\left(x, z_{2}\right) \beta(x) \mathrm{d} x \\
& +\left(R\left(z_{1}\right)-r_{0}\right)\left(P_{0}^{(2)}\left(z_{2}\right)+Q\right) K\left(z_{2}\right) .
\end{aligned}
$$

Similarly, on performing $\sum_{n=0}^{\infty}(4.16) z_{2}^{n}$ and using (3.1), we obtain

$$
V_{0}\left(0, z_{2}\right)=p \int_{0}^{\infty} P_{0}^{(1)}\left(x, z_{2}\right) \mu(x) \mathrm{d} x .
$$

Now, we integrate (4.19) from 0 to $x$ and obtain

$$
P^{(1)}\left(x, z_{1}, z_{2}\right)=P^{(1)}\left(0, z_{1}, z_{2}\right) \exp \left[-\lambda_{1}\left(1-z_{1}\right) x-\lambda_{2}\left(1-z_{2}\right) x-\int_{0}^{x} \mu(t) \mathrm{d} t\right],
$$

where $P^{(1)}\left(0, z_{1}, z_{2}\right)$ is given by (4.27).

Similarly, on integrating, (4.22) gives

$$
V\left(x, z_{1}, z_{2}\right)=V\left(0, z_{1}, z_{2}\right) \exp \left[-\lambda_{1}\left(1-z_{1}\right) x-\lambda_{2}\left(1-z_{2}\right) x-\int_{0}^{x} \beta(t) \mathrm{d} t\right] .
$$

However, by its definition, $V\left(0, z_{1}, z_{2}\right)=V_{0}\left(0, z_{2}\right)$ and, therefore, (4.30) is re-written as

$$
V\left(x, z_{1}, z_{2}\right)=V_{0}\left(0, z_{2}\right) \exp \left[-\lambda_{1}\left(1-z_{1}\right) x-\lambda_{2}\left(1-z_{2}\right) x-\int_{0}^{x} \beta(t) \mathrm{d} t\right]
$$


where $V_{0}\left(0, z_{2}\right)$ is given by (4.28).

Once again integrating (4.29) and (4.31) with respect to $x$ by parts and using (2.2) and (2.4), we have

$$
\begin{gathered}
P^{(1)}\left(z_{1}, z_{2}\right)=P^{(1)}\left(0, z_{1}, z_{2}\right)\left[\frac{1-B^{*}\left[\lambda_{1}\left(1-z_{1}\right)+\lambda_{2}\left(1-z_{2}\right)\right]}{\lambda_{1}\left(1-z_{1}\right)+\lambda_{2}\left(1-z_{2}\right)}\right], \\
V\left(z_{1}, z_{2}\right)=V_{0}\left(0, z_{2}\right)\left[\frac{1-V^{*}\left[\lambda_{1}\left(1-z_{1}\right)+\lambda_{2}\left(1-z_{2}\right)\right]}{\lambda_{1}\left(1-z_{1}\right)+\lambda_{2}\left(1-z_{2}\right)}\right],
\end{gathered}
$$

where

$B^{*}\left[\lambda_{1}\left(1-z_{1}\right)+\lambda_{2}\left(1-z_{2}\right)\right]=\int_{0}^{\infty} \mathrm{e}^{-\left[\lambda_{1}\left(1-z_{1}\right)+\lambda_{2}\left(1-z_{2}\right)\right]} \mathrm{d} B(x)$ is the LST of the service time of a priority unit and $V^{*}\left[\lambda_{1}\left(1-z_{1}\right)+\lambda_{2}\left(1-z_{2}\right)\right]=\int_{0}^{\infty} \mathrm{e}^{-\left[\lambda_{1}\left(1-z_{1}\right)+\lambda_{2}\left(1-z_{2}\right)\right]} \mathrm{d} V(x)$ is the LST of the server's vacation time respectively.

Now, Equation (4.18) can be re-written as

$$
\frac{\mathrm{d}}{\mathrm{d} x} P_{0}^{(1)}\left(x, z_{2}\right)+\left(\lambda_{1}+\lambda_{2}\left(1-z_{2}\right)+\mu(x)\right) P_{0}^{(1)}\left(x, z_{2}\right)=0 .
$$

which, on integration, gives

$$
P_{0}^{(1)}\left(x, z_{2}\right)=P_{0}^{(1)}\left(0, z_{2}\right) \exp \left[-\lambda_{1} x-\lambda_{2}\left(1-z_{2}\right) x-\int_{0}^{x} \mu(t) \mathrm{d} t\right] .
$$

and (4.21) yields

$$
V_{0}\left(x, z_{2}\right)=V_{0}\left(0, z_{2}\right) \exp \left[-\lambda_{1} x-\lambda_{2}\left(1-z_{2}\right) x-\int_{0}^{x} \beta(t) \mathrm{d} t\right] .
$$

Next, we shall determine the integrals

$\int_{0}^{\infty} P^{(1)}\left(x, z_{1}, z_{2}\right) \mu(x) \mathrm{d} x, \int_{0}^{\infty} P_{0}^{(1)}\left(x, z_{2}\right) \mu(x) \mathrm{d} x$ and $\int_{0}^{\infty} V_{0}\left(x, z_{2}\right) \mu(x) \mathrm{d} x$ which appear in the right hand sides

$$
\begin{gathered}
\int_{0}^{\infty} P^{(1)}\left(x, z_{1}, z_{2}\right) \mu(x) \mathrm{d} x=B^{*}\left[\lambda_{1}\left(1-z_{1}\right)+\lambda_{2}\left(1-z_{2}\right)\right] P^{(1)}\left(0, z_{1}, z_{2}\right), \\
\int_{0}^{\infty} P_{0}^{(1)}\left(x, z_{2}\right) \mu(x) \mathrm{d} x=B^{*}\left[\lambda_{1}+\lambda_{2}\left(1-z_{2}\right)\right] P_{0}^{(1)}\left(0, z_{2}\right),
\end{gathered}
$$

Similarly, we multiply Equations (4.31) and (4.35) by $\beta(x)$, integrate by parts with respect to $x$ and obtain

$$
\begin{gathered}
\int_{0}^{\infty} V\left(x, z_{1}, z_{2}\right) \beta(x) \mathrm{d} x=V^{*}\left[\lambda_{1}\left(1-z_{1}\right)+\lambda_{2}\left(1-z_{2}\right)\right] V_{0}\left(0, z_{2}\right) \\
\int_{0}^{\infty} V_{0}\left(x, z_{2}\right) \beta(x) \mathrm{d} x=V^{*}\left[\lambda_{1}+\lambda_{2}\left(1-z_{2}\right)\right] V_{0}\left(0, z_{2}\right) .
\end{gathered}
$$

Using Equations (4.36) to (4.39) into Equations (4.24), (4.27) and (4.28), we obtain

$$
\left(z_{2}-r_{0} K\left(z_{2}\right)\right) P_{0}^{(2)}\left(z_{2}\right)=(1-p) B^{*}\left[\lambda_{1}+\lambda_{2}\left(1-z_{2}\right)\right] P_{0}^{(1)}\left(0, z_{2}\right)+Q r_{0} K\left(z_{2}\right)-Q+V^{*}\left[\lambda_{1}+\lambda_{2}\left(1-z_{2}\right)\right] V_{0}\left(0, z_{2}\right)
$$




$$
\begin{gathered}
z_{1} P^{(1)}\left(0, z_{1}, z_{2}\right)=B^{*}\left[\lambda_{1}\left(1-z_{1}\right)+\lambda_{2}\left(1-z_{2}\right)\right] P^{(1)}\left(0, z_{1}, z_{2}\right)-B^{*}\left[\lambda_{1}+\lambda_{2}\left(1-z_{2}\right)\right] P_{0}^{(1)}\left(0, z_{2}\right) \\
+V^{*}\left[\lambda_{1}\left(1-z_{1}\right)+\lambda_{2}\left(1-z_{2}\right)\right] V_{0}\left(0, z_{2}\right)-V^{*}\left[\lambda_{1}+\lambda_{2}\left(1-z_{2}\right)\right] V_{0}\left(0, z_{2}\right) \\
+\left(R\left(z_{1}\right)-r_{0}\right)\left(P_{0}^{(2)}\left(z_{2}\right)+Q\right) K\left(z_{2}\right) . \\
V_{0}\left(0, z_{2}\right)=p B^{*}\left[\lambda_{1}+\lambda_{2}\left(1-z_{2}\right)\right] P_{0}^{(1)}\left(0, z_{2}\right)
\end{gathered}
$$

Next, we substitute the value of $V_{0}\left(0, z_{2}\right)$ from Equation (4.42) into Equations (4.40) and (4.41), replace

$R\left(z_{1}\right)$ by $\mathrm{e}^{-\lambda_{1} d\left(1-z_{1}\right)}$ and $K\left(z_{2}\right)$ by $\mathrm{e}^{-\lambda_{2} d\left(1-z_{2}\right)}$ from (3.1f) and (3.1g) and simplify. We obtain

$$
\begin{aligned}
\left(z_{2}-r_{0} \mathrm{e}^{-\lambda_{2} d\left(1-z_{2}\right)}\right) P_{0}^{(2)}\left(z_{2}\right)=\left[(1-p)+p V^{*}\left[\lambda_{1}+\lambda_{2}\left(1-z_{2}\right)\right]\right] B^{*}\left[\lambda_{1}+\lambda_{2}\left(1-z_{2}\right)\right] P_{0}^{(1)}\left(0, z_{2}\right)+Q r_{0} \mathrm{e}^{-\lambda_{2} d\left(1-z_{2}\right)}-Q \\
{\left[z-B^{*}\left[\lambda_{1}\left(1-z_{1}\right)+\lambda_{2}\left(1-z_{2}\right)\right]\right] P^{(1)}\left(0, z_{1}, z_{2}\right) } \\
=-B^{*}\left[\lambda_{1}+\lambda_{2}\left(1-z_{2}\right)\right] P_{0}^{(1)}\left(0, z_{2}\right)+p B^{*}\left[\lambda_{1}+\lambda_{2}\left(1-z_{2}\right)\right] V^{*}\left[\lambda_{1}\left(1-z_{1}\right)+\lambda_{2}\left(1-z_{2}\right)\right] P_{0}^{(1)}\left(0, z_{2}\right) \\
\quad-p B^{*}\left[\lambda_{1}+\lambda_{2}\left(1-z_{2}\right)\right] V^{*}\left[\lambda_{1}+\lambda_{2}\left(1-z_{2}\right)\right] P_{0}^{(1)}\left(0, z_{2}\right)+\left(\mathrm{e}^{-\lambda_{11} d\left(1-\lambda_{11}\right)}-r_{0}\right)\left(P_{0}^{(2)}\left(z_{2}\right)+Q\right) \mathrm{e}^{-\lambda_{2} d\left(1-z_{2}\right)}
\end{aligned}
$$

Now, substituting for $P^{(1)}\left(0, z_{1}, z_{2}\right)$ from Equation (4.44) into Equation (4.32), we have

$$
\begin{aligned}
P^{(1)}\left(z_{1}, z_{2}\right)= & \frac{\left(\frac{1-B^{*}\left[\lambda_{1}\left(1-z_{1}\right)+\lambda_{2}\left(1-z_{2}\right)\right]}{\lambda_{1}\left(1-z_{1}\right)+\lambda_{2}\left(1-z_{2}\right)}\right)\left\langle\left(\mathrm{e}^{-\lambda_{1} d\left(1-z_{1}\right)}-r_{0}\right)\left(P_{0}^{(2)}\left(z_{2}\right)+Q\right) \mathrm{e}^{-\lambda_{2} d\left(1-z_{2}\right)}\right\rangle}{\left(z_{1}-B^{*}\left[\lambda_{1}\left(1-z_{1}\right)+\lambda_{2}\left(1-z_{2}\right)\right]\right)} \\
& -\frac{\left(\frac{1-B^{*}\left[\lambda_{1}\left(1-z_{1}\right)+\lambda_{2}\left(1-z_{2}\right)\right]}{\lambda_{1}\left(1-z_{1}\right)+\lambda_{2}\left(1-z_{2}\right)}\right)\left\langle B^{*}\left[\lambda_{1}+\lambda_{2}\left(1-z_{2}\right)\right] P_{0}^{(1)}\left(0, z_{2}\right)\right\rangle}{\left(z_{1}-B^{*}\left[\lambda_{1}\left(1-z_{1}\right)+\lambda_{2}\left(1-z_{2}\right)\right]\right)} \\
& +\frac{\left(\frac{1-B^{*}\left[\lambda_{1}\left(1-z_{1}\right)+\lambda_{2}\left(1-z_{2}\right)\right]}{\lambda_{1}\left(1-z_{1}\right)+\lambda_{2}\left(1-z_{2}\right)}\right)\left\langle p B^{*}\left[\lambda_{1}+\lambda_{2}\left(1-z_{2}\right)\right] V^{*}\left[\lambda_{1}\left(1-z_{1}\right)+\lambda_{2}\left(1-z_{2}\right)\right] P_{0}^{(1)}\left(0, z_{2}\right)\right\rangle}{\left(z_{1}-B^{*}\left[\lambda_{1}\left(1-z_{1}\right)+\lambda_{2}\left(1-z_{2}\right)\right]\right)} \\
& -\frac{\left(\frac{1-B^{*}\left[\lambda_{1}\left(1-z_{1}\right)+\lambda_{2}\left(1-z_{2}\right)\right]}{\lambda_{1}\left(1-z_{1}\right)+\lambda_{2}\left(1-z_{2}\right)}\right)\left\langle p B^{*}\left[\lambda_{1}+\lambda_{2}\left(1-z_{2}\right)\right] V^{*}\left[\lambda_{1}+\lambda_{2}\left(1-z_{2}\right)\right] P_{0}^{(1)}\left(0, z_{2}\right)\right\rangle}{\left(z_{1}-B^{*}\left[\lambda_{1}\left(1-z_{1}\right)+\lambda_{2}\left(1-z_{2}\right)\right]\right)}
\end{aligned}
$$

We have yet to determine the 3 unknowns $P_{0}^{(1)}\left(0, z_{2}\right)$, $P_{0}^{(2)}\left(z_{2}\right)$ and $Q$ appearing in the numerator of the right hand side of (4.45). For this purpose, we proceed as follows.

It can be easily shown that the denominator of the

$$
\begin{aligned}
& \left\langle\left(\mathrm{e}^{-\lambda_{1} d(1-\alpha)}-r_{0}\right)\left(P_{0}^{(2)}\left(z_{2}\right)+Q\right) \mathrm{e}^{-\lambda_{2} d\left(1-z_{2}\right)}\right\rangle-\left\langle B^{*}\left[\lambda_{1}+\lambda_{2}\left(1-z_{2}\right)\right] P_{0}^{(1)}\left(0, z_{2}\right)\right\rangle \\
& +\left\langle p B^{*}\left[\lambda_{1}+\lambda_{2}\left(1-z_{2}\right)\right] V^{*}\left[\lambda_{1}(1-\alpha)+\lambda_{2}\left(1-z_{2}\right)\right] P_{0}^{(1)}\left(0, z_{2}\right)\right\rangle \\
& -\left\langle p B^{*}\left[\lambda_{1}+\lambda_{2}\left(1-z_{2}\right)\right] V^{*}\left[\lambda_{1}+\lambda_{2}\left(1-z_{2}\right)\right] P_{0}^{(1)}\left(0, z_{2}\right)\right\rangle=0 .
\end{aligned}
$$

Now, we solve Equations (4.43) and (4.46) for the two unknowns $P_{0}^{(1)}\left(0, z_{2}\right)$ and $P_{0}^{(2)}\left(z_{2}\right)$. Thus we obtain

$$
P_{0}^{(1)}(o, z)=\frac{\left(\mathrm{e}^{-\lambda_{1} d(1-\alpha)}-r_{0}\right) \mathrm{e}^{-\lambda_{2} d\left(1-z_{2}\right)}\left(1-z_{2}\right) Q}{D(z)}
$$




$$
P_{0}^{(2)}\left(z_{2}\right)=\frac{B^{*}\left[\lambda_{1}+\lambda_{2}\left(1-z_{2}\right)\right]\left\langle\begin{array}{l}
{\left[(1-p)+p V^{*}\left[\lambda_{1}+\lambda_{2}\left(1-z_{2}\right)\right]\right]\left(r_{0}-\mathrm{e}^{-\lambda_{1} d(1-\alpha)}\right) \mathrm{e}^{-\lambda_{2} d\left(1-z_{2}\right)}} \\
-\left[\left(1+p V^{*}\left[\lambda_{1}\left(1-z_{1}\right)+\lambda_{2}\left(1-z_{2}\right)\right]-p V^{*}\left[\lambda_{1}+\lambda_{2}\left(1-z_{2}\right)\right]\right]\left[1-r_{0} \mathrm{e}^{-\lambda_{2} d\left(1-z_{2}\right)}\right]\right.
\end{array}\right) Q}{D(z)}
$$

where $D(z)$ in Equations (4.47) and (4.48) is the common denominator given by

$$
\begin{aligned}
D(z)= & B^{*}\left[\lambda_{1}+\lambda_{2}\left(1-z_{2}\right)\right]\left(\left[(1-p)+p V^{*}\left[\lambda_{1}+\lambda_{2}\left(1-z_{2}\right)\right]\left[\mathrm{e}^{-\lambda_{1} d(1-\alpha)}-r_{0}\right]\right.\right. \\
& -\left[\left(1+\mathrm{p} V^{*}\left[\lambda_{1}\left(1-z_{1}\right)+\lambda_{2}\left(1-z_{2}\right)\right]-p V^{*}\left[\lambda_{1}+\lambda_{2}\left(1-z_{2}\right)\right]\right]\left[z_{2}-r_{0} \mathrm{e}^{-\lambda_{2} d\left(1-z_{2}\right)}\right]\right)
\end{aligned}
$$

Then, we substitute for $P_{0}^{(1)}\left(0, z_{2}\right)$ and $P_{0}^{(2)}\left(z_{2}\right)$ from (4.47) and (4.48) into Equation (4.45) giving us $P^{(1)}\left(z_{1}, z_{2}\right)$. Finally, we shall use the normalizing condition $P^{(1)}(1,1)+P_{0}^{(2)}(1)+Q=1$ to determine the only re- maining unknown $Q$.

Using L' Hopital's rule and proceeding as in Madan and Aby-Dayyeah (2003), we obtain

$$
Q=\frac{\left[1-\lambda_{1}((E(S)+p E(V))]\left(1-\lambda_{2} d\right)\right.}{\lambda_{1} d(E(S)+p E(V))\left(1-\lambda_{2} d\right)+\left[1-\lambda_{1}(E(S)+p E(V))\right]}
$$

where $E(S)$ is the mean service time of a priority unit and $E(V)$ is the mean vacation time of the server.

Having thus determined the value of $Q$, the probability that the server is idle, we have completely determined $P^{(1)}\left(z_{1}, z_{2}\right)$.

Further, system's utilization factor is given by

$$
\rho=1-Q=\frac{\lambda_{1} d\left(( E ( S ) + p E ( V ) ) ( 1 - \lambda _ { 2 } d ) \left[1-\lambda_{1}((E(S)+p E(V))]\left(\lambda_{2} d\right)\right.\right.}{\lambda_{1} d(E(S)+p E(V))\left(1-\lambda_{2} d\right)+\left[1-\lambda_{1}(E(S)+p E(V))\right]}
$$
by

The stability condition, under which the steady state exists, emerges from (4.50 and (4.51)). This condition is given

$$
0<\frac{\lambda_{1} d\left(( E ( S ) + p E ( V ) ) ( 1 - \lambda _ { 2 } d ) \left[1-\lambda_{1}((E(S)+p E(V))]\left(\lambda_{2} d\right)\right.\right.}{\lambda_{1} d(E(S)+p E(V))\left(1-\lambda_{2} d\right)+\left[1-\lambda_{1}(E(S)+p E(V))\right]}<1
$$

Note that (4.52) essentially implies that $\lambda_{1}(E(S)+p E(V))<1$ and $\lambda_{2} d<1$ should jointly hold for the steady state to exist. This is also intuitively true.

\section{Particular Cases}

Case 1: If there are no server vacations, then we let $p=0$ in the above results (4.45) to (4.52) and obtain

$$
\begin{aligned}
& P^{(1)}\left(z_{1}, z_{2}\right)= \frac{\left(\frac{1-B^{*}\left[\lambda_{1}\left(1-z_{1}\right)+\lambda_{2}\left(1-z_{2}\right)\right]}{\lambda_{1}\left(1-z_{1}\right)+\lambda_{2}\left(1-z_{2}\right)}\right)\left\langle\left(\mathrm{e}^{-\lambda_{1} d\left(1-z_{1}\right)}-r_{0}\right)\left(P_{0}^{(2)}\left(z_{2}\right)+Q\right) \mathrm{e}^{-\lambda_{2} d\left(1-z_{2}\right)}\right\rangle}{\left(z_{1}-B^{*}\left[\lambda_{1}\left(1-z_{1}\right)+\lambda_{2}\left(1-z_{2}\right)\right]\right)} \\
&-\frac{\left(\frac{1-B^{*}\left[\lambda_{1}\left(1-z_{1}\right)+\lambda_{2}\left(1-z_{2}\right)\right]}{\lambda_{1}\left(1-z_{1}\right)+\lambda_{2}\left(1-z_{2}\right)}\right)\left\langle B^{*}\left[\lambda_{1}+\lambda_{2}\left(1-z_{2}\right)\right] P_{0}^{(1)}\left(0, z_{2}\right)\right\rangle}{\left(z_{1}-B^{*}\left[\lambda_{1}\left(1-z_{1}\right)+\lambda_{2}\left(1-z_{2}\right)\right]\right)} \\
&\left\langle\left(\mathrm{e}^{-\lambda_{1} d(1-\alpha)}-r_{0}\right)\left(P_{0}^{(2)}\left(z_{2}\right)+Q\right) \mathrm{e}^{-\lambda_{2} d\left(1-z_{2}\right)}\right\rangle-\left\langle B^{*}\left[\lambda_{1}+\lambda_{2}\left(1-z_{2}\right)\right] P_{0}^{(1)}\left(0, z_{2}\right)\right\rangle
\end{aligned}
$$




$$
\begin{gathered}
P_{0}^{(1)}(o, z)=\frac{\left(\mathrm{e}^{-\lambda_{1} d(1-\alpha)}-r_{0}\right) \mathrm{e}^{-\lambda_{2} d\left(\left(1-z_{2}\right)\right.}\left(1-z_{2}\right) Q}{B^{*}\left[\lambda_{1}+\lambda_{2}\left(1-z_{2}\right)\right]\left(\left[z_{2}-r_{0} \mathrm{e}^{-\lambda_{2} d\left(1-z_{2}\right)}\right]\right)} \\
P_{0}^{(2)}\left(z_{2}\right)=\frac{B^{*}\left[\lambda_{1}+\lambda_{2}\left(1-z_{2}\right)\right]\left\langle\left(r_{0}-\mathrm{e}^{-\lambda_{1} d(1-\alpha)}\right) \mathrm{e}^{-\lambda_{2} d\left(1-z_{2}\right)}-\left[1-r_{0} \mathrm{e}^{-\lambda_{2} d\left(1-z_{2}\right)}\right]\right\rangle Q}{B^{*}\left[\lambda_{1}+\lambda_{2}\left(1-z_{2}\right)\right]\left(\left[z_{2}-r_{0} \mathrm{e}^{-\lambda_{2} d\left(1-z_{2}\right)}\right]\right)}
\end{gathered}
$$

We further obtain $Q$ the steady state probability that thw server is idle as

$$
Q=\frac{\left[1-\lambda_{1} E(S)\right]\left(1-\lambda_{2} d\right)}{\lambda_{1} d E(S)\left(1-\lambda_{2} d\right)+\left[1-\lambda_{1} E(S)\right]}
$$

where $\mathrm{E}(\mathrm{S})$ is the mean service time of a priority unit.

The utilization factor of the system is given by

$$
\rho=1-Q=\frac{\lambda_{1} d E(S)\left(1-\lambda_{2} d\right)\left[1-\lambda_{1} E(S)\right]\left(\lambda_{2} d\right)}{\lambda_{1} d E(S)\left(1-\lambda_{2} d\right)+\left[1-\lambda_{1} E(S)\right]}
$$

The stability condition, under which the steady state exists, emerges from (4.57 and (4.58). This condition is given by

$$
0<\frac{\lambda_{1} d E(S)\left(1-\lambda_{2} d\right)\left[1-\lambda_{1} E(S)\right]\left(\lambda_{2} d\right)}{\lambda_{1} d E(S)\left(1-\lambda_{2} d\right)+\left[1-\lambda_{1} E(S)\right]}<1 .
$$

All results in (4.53) to (4.59) agree with the results of Madan and Abu-Dayyeah [15].

We may point out that with suitable substitutions, the main results of this paper will reduce to many other particular cases including a combination of $M / E_{k} / 1$ and $M / D / 1$ queues, a combination of $M / M / 1$ and $M / D / 1$ queues, the case when no priority units arrive at the system and the case when no non-priority units arrive at the system. Further, with $p=0$, the results of all the particular cases of this paper agree with the corresponding particular cases of Madan and Abu-Dayyeah [9].

\section{References}

[1] A. Cobham, "Priority Assignments in Waiting Line Problems," Operions Research, Vol. 2, No. 1, 1954, pp. 70-76. doi:10.1287/opre.2.1.70

[2] T. E. Phipps, "Machine Repair as a Priority Waiting Line Problem," Operations Research, Vol. 4, No. 1, 1956, pp. 76-85. doi:10.1287/opre.4.1.76

[3] L. E. Schrage, "The Queue M/G/1 with Feedback to Lower Priority Queues," Management Science, Vol. 13, No. 7, 1967, pp. 466-474. doi:10.1287/mnsc.13.7.466

[4] N. K. Jaiswal, "Priority Queues," Academic Press, New York, 1968.

[5] K. C. Madan, "A Priority Queueing System with Service Interruptions," Statistica Neerlandica, Vol. 27, No. 3,
1973, pp. $115-123$.

doi:10.1111/j.1467-9574.1973.tb00217.x

[6] B. Simon, "Priorty Queues with Feedback," Journal of the Association for Computing Machinery, Vol. 31, No. 1, 1984, pp. 134-149.

[7] H. Takagi, "Vacation and Priority Systems," Queueing Analysis, Vol. 1, Amsterdam, 1991.

[8] B. D. Choi, and Y. Chang, "Single Server Retrial Queues with Priority Calls," Mathematical and Computer Modeling Vol. 30, No. 3-4, 1999, pp. 7-32. doi:10.1016/S0895-7177(99)00129-6

[9] K. C. Madan and W. Abu-Dayyeah, "On a Combination of M/G/1 and M/D/1 Queues in Non-Preemptive Priority Queueing System," Far East Journal of Theoretical Statistics, Vol. 10, No. 2, 2003, pp. 133-146.

[10] U. N. Bhat, "Elements of Applied Stochastic Processes," Wiley, New York, 1972.

[11] Y. Levy and U. Yechiali, "Utilization of Idle Time in an M/G/1 Queueing System," Management Science, Vol. 22, No. 2, 1975, pp. 202-211. doi:10.1287/mnsc.22.2.202

[12] L. Kleinrock, "Queueing Systems, Vol. 2, Computer Applications," Wiley, New York, 1976.

[13] J. W. Cohen, "The Single Server Queue," 2nd Edition, North-Holland, Amsterdam, 1982.

[14] T. T. Lee, "M/G/1/N Queue with Vacation Times and Exhaustive Service Discipline," Operations Research, Vol. 32, No. 4, 1984, pp. 774-786. doi:10.1287/opre.32.4.774

[15] D. Gross and C. M. Harris, "Fundamentals of Queueing Theory," 2nd Edition, Wiley, New York, 1985.

[16] D. R. Cox and H. D. Miller, "The Theory of Stochastic Processes," Chapman and Hall, London, 1994.

[17] H. C. Tijms, "Stochastic Models: An Algorithmic Approach," Wiley, New York, 1994.

[18] T. Yang and H. Li, "The M/G/1 Retrial Queue with the Server Subject to Starting Failures," Queueing Systems, Vol. 16, No. 1-2, 1994, pp. 83-96. doi:10.1007/BF01158950

[19] B. D. Bunday, "Basic Queueing Theory," 2nd Edition, Edward Arnold, Melbourne, 1995.

[20] K. C. Madan, "An M/G/1 Queue with Optional Deterministic Server Vacations," Metron, Vol. 57, No. 3-4, 1999, pp. 83-95.

[21] K. C. Madan, “An M/G/1 Queue with Second Optional Service," Queueing Systems, Vol. 34, No. 1-4, 2000, pp. 37-46. doi:10.1023/A:1019144716929 